\title{
DESIGUALDADES SOCIALES EN LA ASOCIACIÓN ENTRE VIVIR EN PAREJA/ ESTAR CASADO Y LA SALUD ENTRE TRABAJADORES EN ESPAÑA
}

\author{
LUCÍA ARTAZCOZ \\ Agència de Salut Pública. Barcelona
}

Recibido/15/07/2011

Aceptado/25/09/2011

\section{Referencia del artículo comentado}

ARTAZCOZ, Lucia; CORTÉS, Inmaculada; BORRELL, Carmen; ESCRIBÀAGÜIR, Vicenta y CASCANT Lorena. Social inequalities in the association between partner/marital status and health among workers in Spain. Soc Sci E Med 2011; 72: 600-607.

\section{Resumen}

Los objetivos de este estudio son examinar la asociación entre el estado marital o de unión con una pareja y varios temas de salud entre trabajadores y determinar si depende del género y la clase social ocupacional. La muestra estaba compuesta por todos los trabajadores de 21 a 64 años entrevistados en la Encuesta Nacional de Salud de 2006 (8563 hombres y 5881 mujeres). El estado marital o de unión con una pareja estaba compuesto por 7 categorías: casado/a y viviendo con su esposa/o (categoría de referencia), casado/a sin vivir con su esposa/o, cohabitando, soltero/a y viviendo con sus padres, soltero/a sin vivir con sus padres, separado/a - divorciado/a y viudo/a. Se analizaron 4 factores relacionados con la salud: salud auto-percibida, salud mental, consumo de fármacos psiquiátricos e hipertensión. Se utilizaron modelos de análisis de regresión logística múltiple estratificados por sexo y clase social. Las mujeres trabajadoras en ocupaciones manuales que cohabitaban reportaron con mayor frecuencia un estado de pobre salud auto-percibida, pobre salud mental, y consumo de fármacos psiquiátricos e hipertensión que las casadas que vivían con sus esposos. En este grupo, la prevalencia de pobre salud fue incluso mayor cuando se compara con las solteras. 
Entre los hombres trabajadores no manuales, ser casado y no vivir con la esposa se asoció con un pobre estado de salud auto-percibida, pobre salud mental e hipertensión. No se detectaron diferencias en salud entre ser casado y el resto de los estados marital o de unión con una pareja para las diferentes combinaciones de género y clase social e, incluso, algunos grupos de solteros reportaron mejor salud que los casados. Nuestros resultados muestran que no hay evidencia que ser casados y vivir con la esposa está inequívocamente relacionado con mayor salud entre los españoles trabajadores. Esto enfatiza la importancia de no solamente considerar el estado marital, si no el de convivencia en pareja, así como los roles de género, clase social, y contexto sociocultural en el análisis de la asociación entre las características familiares y la salud. 


\section{Introducción}

Desde principios de los años 80 numerosos estudios han analizado la relación entre el estado civil y la salud. A diferencia de las publicaciones iniciales que observaban un efecto protector del matrimonio para los hombres pero no para las mujeres, la mayor parte de los estudios recientes documentan un efecto protector para los dos sexos, ${ }^{123}$ aunque en algunos estudios la magnitud de este efecto es superior en los hombres. Se han propuesto dos hipótesis, que no son mutuamente excluyentes, para explicar estos hallazgos. La «hipótesis de protección» sugiere que el estado civil tiene un impacto causal sobre la salud debido a una variedad de factores ambientales, sociales y psicológicos. Por ejemplo, el matrimonio podría aumentar el apoyo social y los ingresos y reducir las conductas de riesgo. Por el contrario, la «hipótesis de selección» sostiene que la salud y las conductas relacionadas con la salud determinan el estado civil o de convivencia en primer lugar. Por lo tanto los individuos sanos tienen más probabilidades de vivir en pareja.

La mayoría de los estudios publicados hasta ahora se refieren a la situación en Estados Unidos, Canadá, países del norte de Europa o Reino Unido, pero poco se sabe sobre los países mediterráneos que son muy diferentes a los anglosajones en relación con la familia y las formas de convivencia. Este es el primer artículo que analiza la relación entre el estado civil o de convivencia y la salud en España y trata de superar algunas de las limitaciones metodológicas de estudios anteriores. Examina cuatro indicadores de salud: salud percibida, salud mental, consumo de psicofármacos e hipertensión arterial. Sorprendentemente los resultados son muy distintos a los publicados hasta ahora.

1. Lillard LA, Waite LJ. Til death do us part: Marital disruption and mortality. Am J Sociol 1995; 100: 1131-1156.

2. Hu Y., Goldman N. Mortality differentials by marital status: An international comparison. Demography 1990; 27, 233-250.

3. Cheung YB. Marital status and mortality in British women: A longitudinal study. Int J Epidemiol. 2000; 29: 93-99.

Feminismo/s 18, diciembre 2011, pp. 301-308 


\section{Superando limitaciones metodológicas de la investigación previa}

Este artículo plantea varias limitaciones metodológicas o conceptuales de estudios previos que se señalan a continuación:

1.- ¿Estar casado o vivir en pareja sin estarlo es similar y tiene igual impacto en la salud?

Aunque muchos estudios han considerado como una única categoría estar casado/a o vivir en pareja, (de hecho muchas encuestas de salud recogen esta información en una única categoría), estudios recientes apuntan a que la salud de las personas que viven en pareja sin estar casadas es peor que la de las casadas, pero mejor que el resto de las situaciones de estado civil o de convivencia. Se han señalado varias razones. En primer lugar, las parejas de hecho pueden ser diferentes en las actitudes y comportamientos de las que se casan. En segundo lugar, porque las convivencias sin estar casados/as suelen ser de menor duración que los matrimonios, y por lo tanto las parejas que cohabitan pueden no tener los beneficios en salud a largo plazo que se encuentran en el matrimonio. En tercer lugar, los acuerdos económicos dentro del matrimonio y dentro de la convivencia pueden ser diferentes. Para algunos, la cohabitación proporciona independencia económica dentro de una relación íntima, lo que combina los beneficios de la soltería con los de una unión sexual.

2.- ¿Cómo evitar o reducir un sesgo de causalidad inversa en estudios sobre este tema realizados con diseños transversales?

El matrimonio puede proporcionar beneficios pero también podría suceder que las personas sanas tuvieran más probabilidad de encontrar pareja, es decir, que los resultados se explicasen por un sesgo de causalidad inversa. El mismo sesgo existiría entre las personas separadas o divorciadas, ya que se ha observado que el mal estado de salud puede ser la causa de la separación o el divorcio. Este estudio, realizado con datos de una encuesta de diseño transversal, reduce este posible sesgo seleccionando sólo población trabajadora en la que también existe un sesgo de selección, el sesgo del trabajador sano. Por tanto, se analiza una población más sana que la general en la que no están personas, que por razones de salud no trabajan o que por las mismas razones tengan dificultades para encontrar pareja. 
3.- ¿Es necesario ajustar por el tipo de contrato?

Es necesario ajustar por una variable si ésta es una posible fuente de confusión. El tipo de contrato lo es, ya que se ha observado que está relacionado tanto con el estado de salud como con la probabilidad de estar casado o vivir en pareja. ${ }^{4}$

4.- ¿Cómo se examina en el artículo si la relación entre el estado civil o de convivencia es diferente según el sexo?

Podría suceder que estar casado/a fuera beneficioso para un sexo pero lo fuera menos, o no lo fuera e incluso fuera un factor de riesgo, para el otro. Para examinar esta interacción se separa el análisis por el sexo.

5.- ¿Cómo se justifica en el artículo que la asociación entre el estado civil o de convivencia puede ser diferente según la clase social?

Los patrones de género pueden diferir según la clase social. Es probable que las diferencias de género en el poder entre los miembros de la pareja sean mayores entre las parejas con nivel socioeconómico bajo, debido a actitudes más tradicionales sobre los roles de género entre las personas de bajo nivel de estudios y, por tanto, el potencial beneficio de estar casado o de vivir en pareja, puede ser menor para las mujeres de clases sociales menos privilegiadas. Aunque la mayoría de los estudios previos ajustan el análisis por la posición socioeconómica, examinar la hipótesis anterior requiere separar el análisis por la clase social. En este estudio se analizan dos grupos: trabajadores/as manuales y no manuales.

6.- ¿Por qué los resultados pueden ser diferentes en España que en países anglosajones de similar nivel de desarrollo?

Hay diferencias importantes entre el sur de Europa y otros países desarrollados en términos de matrimonio y convivencia en pareja y por lo tanto la relación entre el estado civil y la salud puede ser diferente. Por ejemplo, los hijos e hijas de los países del sur de Europa en general abandonan el hogar familiar más tarde, y lo hacen con más frecuencia para casarse y menos frecuentemente por razones de empleo o educación superior, en comparación con el norte

4. Artazcoz L, Benach J, Borrell C, Cortès I. Social inequalities in the impact of flexible employment on different domains of psychosocial health. J Epidemiol Community Health 2005; 59: 761-767.

Feminismo/s 18, diciembre 2011, pp. 301-308 
de Europa. ${ }^{5}$ Los adultos jóvenes en Italia, Grecia, España y Portugal se quedan mucho más tiempo con sus padres, especialmente cuando están estudiando, antes de establecerse de forma estable en el mercado laboral o en una relación de pareja estable. ${ }^{6}$ El alto desempleo y las tasas de inestabilidad laboral entre los adultos jóvenes, la escasez de viviendas en alquiler o una vivienda adecuada para las personas solteras en estos países, así como los factores culturales son algunas de las razones de este patrón del sur de Europa ${ }^{7}$. En estas circunstancias la familia podría tener un efecto protector sobre el estado de salud entre los adultos jóvenes. Sin embargo, hasta ahora no se han publicado estudios examinando esta cuestión. Además, la cohabitación es menos frecuente que en los países del norte europeo.

\section{Reflexiones sobre los resultados más importantes}

1.- A diferencia de la mayoría de estudios previos, en este estudio estar casado, en general, no se asocia con mejores indicadores de salud que el resto de las situaciones de estado civil o de convivencia.

Las limitaciones metodológicas de estudios previos podrían explicar por qué se encuentran muy pocas diferencias en el estado de salud según el estado civil y además en algunos grupos las personas solteras tienen mejor estado de salud que las casadas. En los países del sur de Europa una característica especial es el carácter selectivo del abandono del hogar de los padres por parte de los adultos jóvenes que lo hacen cuando tienen una situación laboral y/o de pareja estable. Por el contrario, en los países nórdicos los adultos jóvenes tienden a separarse de sus padres mucho antes de establecerse en el mercado laboral, con ingresos insuficientes y antes de formar una nueva familia. Por lo tanto, en comparación con el Sur de Europa, en los países nórdicos hay un volumen mucho mayor de adultos jóvenes menores de 30 años, con ingresos marginales, todavía estudiando y que viven en hogares unipersonales. Además la restricción de la población de estudio a personas trabajadoras reduce o elimina el posible sesgo de causalidad inversa existente en estudios transversales previos.

5. Holdsworth C. Leaving home in Britain and Spain. European Sociological Review 2000; $16,201-222$.

6 . Vogel J. European welfare regimes and the transition to adulthood: a comparative and longitudinal perspective. Social Indicators Research 2002; 59, 275-299.

7. Iacovou M. Regional differences in the transition to adulthood. The Annals of the American Academy of Political and Social Sciences 2002; 580, 40-69. 
2.- Entre las trabajadoras manuales se observó una relación consistente entre la cohabitación y los cuatro indicadores de mala salud y las diferencias fueron mayores cuando las parejas de hecho se compararon con los solteros.

Las diferencias en la calidad de las relaciones entre las personas casadas y las que viven en pareja pueden ser mayores, entre las trabajadoras manuales, debido a las desigualdades de poder basadas en la probable relación de género, lo que explicaría el hallazgo de este estudio.

3.- Entre los trabajadores no manuales se encuentra una asociación consistente entre estar casado y no vivir con la pareja, para tres de los cuatro indicadores de mala salud analizados.

Muchos de los trabajadores españoles no manuales que están casados y no conviven con sus parejas podrían estar en las fases iniciales de un proceso de separación o divorcio y por tanto asumiendo el estrés económico propio de estas etapas en la que la mayoría de los hombres suelen perder su vivienda y deben asumir costes económicos relacionados con la ruptura y el mantenimiento de hijos e hijas. La razón por la asociación entre estar casado y no vivir con la pareja se limita a los hombres de clases no manuales lo que podría estar relacionado con las diferencias de clase social en el aumento de la deuda familiar en España en los últimos años. La proporción de familias españolas con una hipoteca fue mayor entre las familias con mayores ingresos ${ }^{8}$. Llama la atención que, aunque el número de personas en esta situación es bajo, las dos ratios son altas y los intervalos de confianza ajustados.

\section{Conclusiones}

Este artículo es un ejemplo de que los determinantes sociales de la salud pueden variar según el tiempo y el lugar analizado. Además, señala la importancia de examinar de manera simultánea las desigualdades de género y de clase social. Con frecuencia, los estudios sobre las desigualdades de clase social en la salud no han tenido en cuenta el papel del género, mientras los de género se han centrado en los determinantes sociales de la salud de las mujeres, enfatizando los roles familiares y considerando en segundo plano el trabajo remunerado o la clase social ocupacional. Finalmente, remarca la importancia

8. Banco de España. Dirección General de Servicios de Estudios. Encuesta Financiera de las Familias 2005. [Bank of Spain. General Directorate of Services of Studies. Family Financial Survey, 2005]. Available at: http://www.bde.es/estadis/eff/Separata_EFF_2007. pdf. Accessed December 12, 2007. Spanish. 
del desarrollo de marcos teóricos que tengan en cuenta los posibles sesgos, variables de confusión y de interacción de manera explícita.

\section{Referencias bibliográficas}

ARTAZCOZ L, BenACH J, BorRell C, CORTÈs I. Social inequalities in the impact of flexible employment on different domains of psychosocial health. J Epidemiol Community Health 2005; 59: 761-767.

BANCO DE España. Dirección General de Servicios de Estudios. Encuesta Financiera de las Familias 2005 http://www.bde.es/estadis/eff/Separata_EFF_2007. pdf. Accessed December 12, 2007. Spanish.

CHEUnG YB. Marital status and mortality in British women: A longitudinal study. Int J Epidemiol. 2000; 29: 93-99.

HOLDSWORTH C. Leaving home in Britain and Spain. European Sociological Review 2000; 16, 201-222.

Hu Y, GOLDMAN N. Mortality differentials by marital status: An international comparison. Demography 1990; 27: 233-250.

IACOVOU M. Regional differences in the transition to adulthood. The Annals of the American Academy of Political and Social Sciences 2002; 580, 40-69.

Lillard LA, WAite LJ. Til death do us part: Marital disruption and mortality. Am J Sociol 1995; 100: 1131-1156.

VOGEL J. European welfare regimes and the transition to adulthood: a comparative and longitudinal perspective. Social Indicators Research 2002; 59, 275-299. 